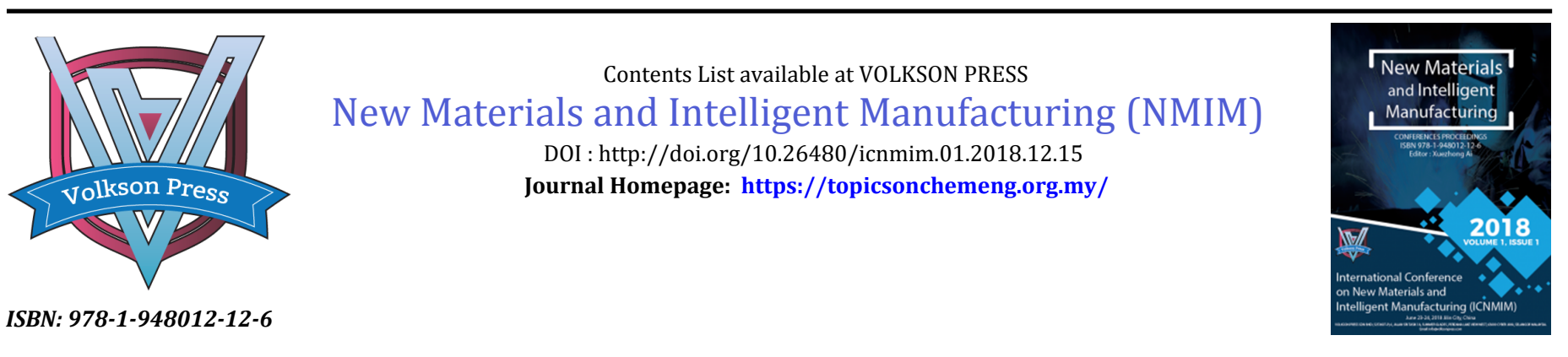

\title{
PREPARATION AND STUDY OF PROPERTIES OF DI(TRIMETHYLOLPROPANE) BIPHOSPHATE ETHANOLAMINE SALT
}

\author{
Wei Jiang ${ }^{1 *}$, Ziyuan $\mathrm{Li}^{2}$ \\ ${ }^{1}$ School of Materials Science and Engineering, Jilin Institute of Chemical Technology Jilin City, Jilin Province 132022 \\ ${ }^{2}$ Postgraduate of Jilin Institute of Chemical Technology Jilin City, Jilin Province 132022 \\ *Corresponding Author Email: jiangweijl@163.com.
}

This is an open access article distributed under the Creative Commons Attribution License, which permits unrestricted use, distribution, and reproduction in any medium, provided the original work is properly cited

\section{ARTICLE DETAILS}

Article History:

Received 26 June 2018

Accepted 2 July 2018

Available online 1 August 2018

\section{ABSTRACT}

A new type of halogen-free flame retardant has been synthesized with raw materials of Di(trimethylolpropane) and phosphorus oxychloride, and the influence of the raw material ratio, reaction temperature and reaction time on the yield has been discussed. Furthermore, the flame-retardant combustion performance has been tested by vertical burning test. The flame-retardant effect and thermal stability of the target product on different fabrics have been investigated, and the structures of the intermediates and the target products were characterized by Fourier transform infrared spectrometer. After discussing the different flame-retardant effects on different fabrics, the conclusions have been drawn: The Di(trimethylolpropane) biphosphate ethanolamine salt has different flame-retardant effects on polyester, acrylic, chinlon, cotton and blended fabrics, of which the flame-retardant effect on chinlon, polyester and cotton is fairly good, but the flame-retardant effect is unremarkable for blended fabrics and acrylic.

\section{KEYWORDS}

Ditrimethylolpropane, preparation, performance, Flame retardant effect, Characterization.

\section{INTRODUCTION}

In recent several decades, the three major synthetic materials and their products have developed rapidly; Furthermore, polymer materials have the characteristics of energy saving, light weight and good processability, therefore, they are rapidly replacing traditional raw materials such as metals, cement and wood, they are widely used in various fields and has obtained significant economic and social benefits [1-4]. However, most polymer materials are easily decomposed and combusted at high temperatures; In the event of a fire, they are ignited in the air [5-9]. Furthermore, their molten droplets may also ignite other combustibles, and as a result, fires are caused and there are serious fire hazards. Flame retardants belong to a class of additives that may prevent or inhibit the combustion of polymers.

The serious drawback of halogen flame retardants is that they generate a large amount of toxic and corrosive gases when they are burned; These can cause corrosion of the circuit system switches and other metal objects and may cause harm to human respiratory and other organs, these are even life-threatening [10-13]. It has become a trend in the field of flame retardant in the world to develop a kind of halogen-free flame retardant with low-toxicity, low-smoke, low-environmental impact and excellent flame- retardant performance to replace the halogen flame retardants. Confront with increasingly stringent environmental and safety requirements, new flame retardants such as those with no toxicity, highefficiency and low-smoke toxicity have become their development direction [14-16].

Therefore, the study of flame retardants has played a crucial role in the development and application of new materials. The future research direction of flame retardants is orientated to halogen-free, highly efficient and environment-friendly types. A new type of halogen-free flame retardant has been synthesized with raw materials of Di (trimethylolpropane) and phosphorus oxychloride; This flame retardant has a large molecular weight, with a stable ring structure; The thermal stability is higher than that of commonly used flame retardants, and the flame-retardant effect is better.

\section{EXPERIMENTAL PART}

\subsection{Main experimental drugs}

The main drugs are Di(trimethylolpropane) (PetroChina Jilin Petrochemical Company); Phosphorus oxychloride (Jihua Group Lianhua Fuli Chemical Factory); Dioxane (Tianjin Damao Chemical Reagent Co., Ltd.); Ethanolamine (Tianjin Yongda Chemical Reagent Co., Ltd.)

\subsection{Experimental principle}

(1) Synthesis of Di(trimethylolpropane)bisphosphate

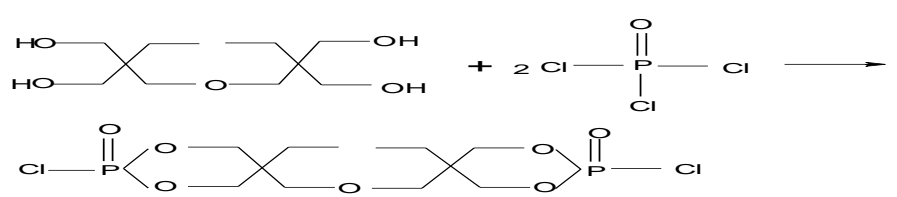

(2) Synthesis of Di(trimethylolpropane) diphosphoryl chloride as the intermediate Hydrolysis of the intermediate to form Di(trimethylolpropane)bisphosphate

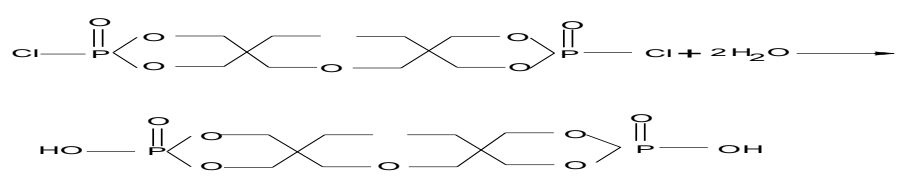


(3) Synthesis of Di(trimethylolpropane) biphosphate ethanolamine salt as the target product

$$
\mathrm{P}_{\mathrm{PO}}^{\mathrm{O}}<\mathrm{O}_{\mathrm{O}}^{\mathrm{O}}>\mathrm{P}-\mathrm{OH}+2 \mathrm{NH}_{2} \mathrm{CH}_{2} \mathrm{CH}_{2} \mathrm{OH} \longrightarrow
$$

$\mathrm{HOCH}_{2} \mathrm{CH}_{2} \mathrm{NH}_{2} \cdot \mathrm{HO}-\stackrel{\mathrm{P}}{\mathrm{P}}<\mathrm{O} \longrightarrow \mathrm{O} \longrightarrow \mathrm{O}_{\mathrm{P}}^{\mathrm{O}}-\mathrm{OH}_{\mathrm{NH}} \mathrm{CH}_{2} \mathrm{CH}_{2} \mathrm{OH}$

\subsection{Experimental procedure}

The experimental procedure was as follows: Assembling, adjusting the experimental device and checking the air tightness. Di(trimethylolpropane) was added to a four-necked flask equipped with a thermometer, stirrer, and spherical condenser tube according to the usage amount; After heating to about $50^{\circ} \mathrm{C}$, phosphorus oxychloride was weighed \& put into a four-necked flask: the stirrer \& condenser were opened and the Di(trimethylolpropane) was weighed and put into the flask. After parker sing, hydrolysis and amination, finally the target product Di(trimethylolpropane) bisphosphonate ethylamine salt was taken out and sealed \& stored. All power supplies and condensate were turned off.

\subsection{Measurement}

Nicolet 6700 type red infrared spectrometer was used to identify the product structure; Furthermore, the flame-retardant combustion performance was tested with fabric vertical burning test; Please refer to GB/T 5455-1997 «Textile burning performance test vertical method» for testing.

\section{RESULTS AND DISCUSSION}

\subsection{Discussion of the synthetic process factors}

\subsubsection{Influence of reaction temperature on the yield}

Other reaction conditions were fixed, i.e., the molar ratio among Di(trimethylolpropane), phosphorus oxychloride and water were 1:4:2, only the reaction temperatures of various steps were changed, thus the experimental results as shown in table 1 were obtained.

Table 1: Influence of reaction temperature on the yield

\begin{tabular}{|cccc|}
\hline No. & $\begin{array}{c}\text { Reaction temperature of the } \\
\text { 1st step }\end{array}$ & $\begin{array}{c}\text { Reaction temperature of the } \\
\text { 2nd step }\end{array}$ & Yield/\% \\
\hline 1 & 60 & 75 & 78.72 \\
2 & 60 & 80 & 80.51 \\
3 & 60 & 85 & 81.25 \\
4 & 70 & 75 & 84.29 \\
5 & 70 & 80 & 86.49 \\
6 & 70 & 85 & 85.64 \\
7 & 80 & 75 & 80.30 \\
9 & 80 & 80 & 86.93 \\
\hline
\end{tabular}

The following conclusions were drawn from table 1: For ethanolamine, the yield is the highest when the phosphating reaction temperature in the 1st step is $70^{\circ} \mathrm{C}$ and the amination reaction temperature in the $3 \mathrm{rd}$ step is 75 $\sim 80^{\circ} \mathrm{C}$.The reason is that there will be more side reactions when the temperature is too high, on the other hand, the reaction proceeds insufficiently, affecting the yield when the temperature is low.

\subsubsection{Influence of phosphating reaction time on the yield of the intermediate}

The following conditions were selected: the reaction temperature was $70^{\circ} \mathrm{C}$, the molar ratio between Di(trimethylolpropane) and phosphorus oxychloride is $1: 4$, the reaction time was regarded as the variable. The results are as shown in Table 4.

Table 4: Influence of phosphating reaction time on the yield of the intermediate

\begin{tabular}{|ccccc|}
\hline No. & $\begin{array}{c}\text { Reaction } \\
\text { time/h }\end{array}$ & Yield/\% & Product color and shape & Cause analysis \\
\hline 1 & 3 & 69.72 & Viscous milk white liquid & Incomplete reaction \\
2 & 4 & 77.41 & Viscous light-yellow paste & $\begin{array}{c}\text { Complete reaction } \\
\text { Complete reaction } \\
\text { Complete reaction, with partial } \\
\text { carbonization }\end{array}$ \\
4 & 5 & 79.52 & Viscous light-yellow paste \\
& 6 & 78.88 & Viscous light red brown paste & \\
\hline
\end{tabular}

The following analysis can be carried out from table 4: The intermediate yield increases with the time; the yield of the intermediate product reaches its maximum at $5 \mathrm{~h}$. When the reaction time continues to increase, side reactions of the intermediates occur, the yield begins to decline, and this is not conducive to the experiment.

\subsection{Infrared spectrum}

The synthesized intermediate and the final product were mixed and ground with $\mathrm{KBr}$ powder, and the infrared spectrogram data was measured after tableting, the test result was shown in figure 1.

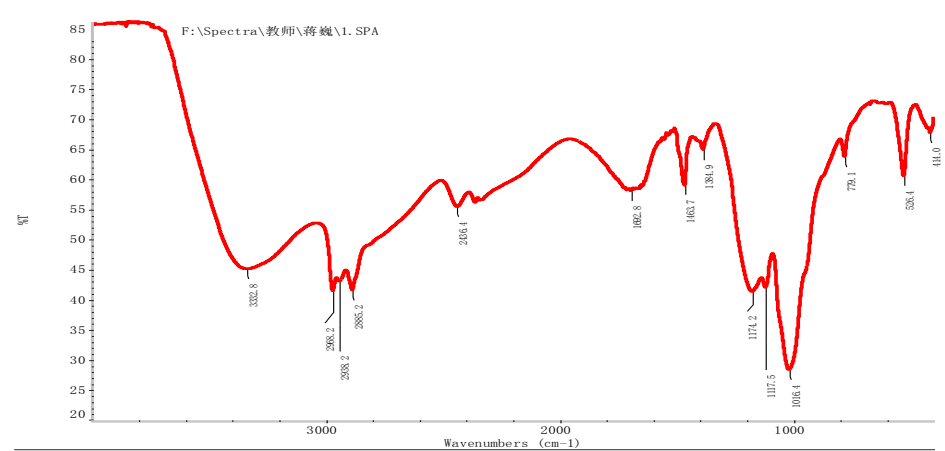

Figure 1: Infrared spectrum of the target product

As shown in figure 1, the wave number $3332.8 \mathrm{~cm}^{-1}$ corresponds to formation of intramolecular hydrogen bond $-\mathrm{OH}$; Due to formation of 
intramolecular hydrogen bond $-\mathrm{OH}$, the reduction of the key force constant occurs, the absorption shifts to lower wave numbers (around $3300 \mathrm{~cm}^{-1}$ ), the peak type is wide and blunt; The wave number of $2965.1 \mathrm{~cm}^{-1}$ corresponds to $-\mathrm{CH}_{3}$, the wave number of $2965.1 \mathrm{~cm}^{-1}$ corresponds to $-\mathrm{CH}_{2}$, the wave number of $2883.4 \mathrm{~cm}^{-1}$ corresponds to carbon-hydrogen bond in $-\mathrm{CH}_{2}-\mathrm{OH}$; Because four absorption peaks are generally visible in the saturated carbon and hydrogen stretching vibrations, among which, $2960 \mathrm{~cm}^{-1}$ and $2870 \mathrm{~cm}^{-1}$ belong to $\mathrm{CH}_{3}, 2925 \mathrm{~cm}^{-}$ ${ }^{1}$ and $2850 \mathrm{~cm}^{-1}$ belong to $\mathrm{CH}_{2}$; When $\mathrm{CH}_{3}$ or $\mathrm{CH}_{2}$ is connected to an oxygen atom, the absorption shifts to lower wave numbers; The wave number of $1169.8 \mathrm{~cm}^{-1}$ corresponds to symmetric vibration of $\mathrm{C}-\mathrm{O}-\mathrm{C}$; The wave number of $1064.4 \mathrm{~cm}^{-1}$ corresponds to P-O-C; The wave number of 550.6 $\mathrm{cm}^{-1}$ corresponds to a moderately divergent double peaks $\mathrm{P}=0$ double bond. To sum up, the synthesized product was indeed Di(trimethylolpropane) diphosphate ethanol amine salt.

\subsection{Discussion of flame retardant effect of the product}

The molar ratio between Di(trimethylolpropane) and phosphorus oxychloride is $1: 4$, phosphating reaction temperature is $70^{\circ} \mathrm{C}$, the reaction time is $5 \mathrm{~h}$, the molar ratio of hydrolysis reaction is $1: 2$, the reaction temperature is $75^{\circ} \mathrm{C}$, the reaction time is $5 \mathrm{~h}$, the molar ratio of amination reaction is $1: 2$, the reaction temperature is $80^{\circ} \mathrm{C}$, the reaction time is $4 \mathrm{~h}$, and the flame-retardant effect of the target product on different fabrics have been investigated.

The flame-retardant effects of the product on polyester, acrylic, cotton, chinlon and blended fabrics are shown in Figures 4-8.

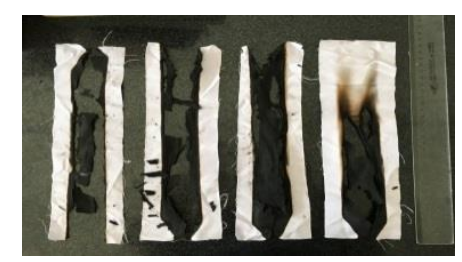

Figure 5: Figure showing flame retardant effect on acrylic

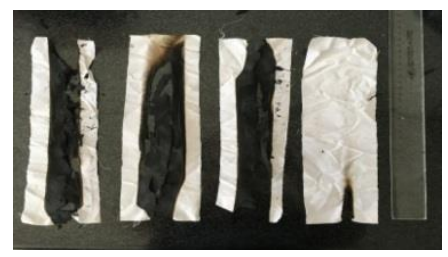

Figure 4: Figure showing flame retardant effect on polyester

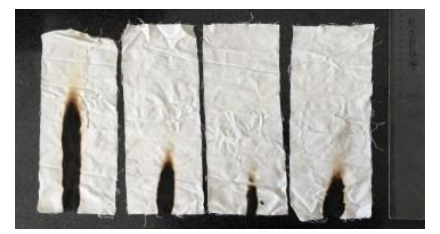

Figure 6: Figure showing flame retardant effect on cotton

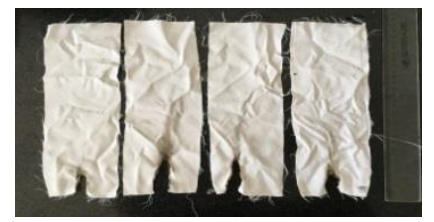

Figure 7: Figure showing flame retardant effect on chinlon

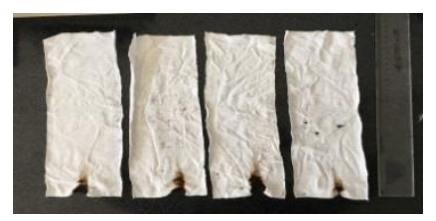

8

Figure 8: Figure showing flame retardant effect on blended fabrics

It can be concluded through comparing the above diagrams that, the intumescent flame retardant has a remarkable flame-retardant effect on cotton, chinlon and blended fabrics, but the flame-retardant effect on polyester and acrylic is not so good. The reason is as follows: Firstly, the microstructure of each fabric is different, and the product flame retardant property of the fabric may be different; Secondly, the same flame retardant has different affinities to different fabrics and shows different flameretardant effects. Therefore, it showed different flame-retardant effects on different fabrics.

\section{CONCLUSION}

Di(trimethylolpropane), phosphorus oxychloride and ethanol amine were used as the main materials in the experiment, and the intumescent flame retardant, Di(trimethylolpropane) biphosphate ethanolamine salt was synthesized, and the flame-retardant effect of the target product on different fabrics have been tested; Furthermore, the following conclusions have been drawn.

(1) The preferable synthesis process of a halogen-free flame retardant is as follows: The molar ratio between Di(trimethylolpropane) and phosphorus oxychloride is $1: 4$, phosphating reaction temperature is $70^{\circ} \mathrm{C}$, the reaction time is $5 \mathrm{~h}$,the molar ratio of hydrolysis reaction is $1: 2$, the reaction temperature is $75^{\circ} \mathrm{C}$, the reaction time is $5 \mathrm{~h}$, the molar ratio of amination reaction is $1: 2$, the reaction temperature is $80^{\circ} \mathrm{C}$, the reaction time is $4 \mathrm{~h}$, and the flame-retardant effect of the target product on different fabrics is fairly good.

(2) After discussing the flame-retardant effects on different fabrics, it has been shown that the intumescent flame retardant has a better flameretardant effect on chinlon, cotton and blended fabrics, but the flameretardant effect on polyester and acrylic is not so good.

(3) Infrared spectroscopic analysis and differential scanning calorimetry have been carried out on the final product, and the structure and thermal properties of the product have been characterized.

\section{ABOUT THE AUTHOR}

About the Author: * Jiangwei (contact of communications) (1970), female, with native place Jilin City, Jilin Province, professor, master tutor, mainly engaged in the study of synthesis and modification of polymer materials.Tel:13843200858.

\section{REFERENCES}

[1] Yang, Z., Wang, X., Lei, D. 2012. A durable flame retardant for cellulosic fabrics [J]. Polymer Degradation and Stability, 97, 2467-2472.

[2] Zhao, C.S., Huang, F.L., Xiong, W.C. 2008. A novel halogen-free flame retardant for glass-fiber-reinforced poly (ethylene terephthalate) [J]. Polymer Degradation and Stability, 93, 1188-1193.

[3] Covaci, A., Voorspoels, S., Abdallah, M.A.E. 2009. Analytical and environmental aspects of the flame retardant tetrabromobisphenol-A and its derivatives [J]. Journal of Chromatography A, 1216, 346-363.

[4] Xing, W.Y., Song, L., Hu, Y. 2013. Combustion and thermal behaviors of the novel UV-cured intumescent flame-retardant coatings containing phosphorus and nitrogen [J]. E-Polymers, 63, 684-694.

[5] Wang, B., Qian, X., Shi, Y. 2015. Cyclodextrin microencapsulated ammonium polyphosphate: Preparation and its performance on the thermal, flame retardancy and mechanical properties of ethylene vinyl acetate copolymer [J]. Composites, 69, 22-30.

[6] Moafi, H.F., Mostashari, S.M. 2014. Flame-resistant polymeric composite fibers based on nanocoating flame retardant: thermogravimetric study and production of á-Al203 nanoparticles by flame combustion [J]. Journal of Polymer Engineering, 34 (9), 803-812.

[7] Jing, J., Zhang, Y., Tang, X. 2017. Layer by layer deposition of polyethylenimine and bio-based polyphosphate on ammonium polyphosphate: A novel hybrid for simultaneously improving the flame retardancy and toughness of polylactic acid [J]. Polymer, 108, 361-371.

[8] Hashim, R., Sulaiman, O., Kumar, R.N. 2009. Physical and mechanical properties of flame retardant urea formaldehyde medium density fiberboard [J]. Journal of Materials Processing Technology, 209, 635-640. 
[9] Cheng, X.W., Guan, J.P., Tang, R.C. 2016. Phytic acid as a bio-based phosphorus flame retardant for poly (lactic acid) nonwoven fabric [J]. Journal of Cleaner Production, 124, 114-119.

[10] Feng, C., Li, Z., Liang, M. 2014. Preparation and characterization of a novel oligomeric charring agent and its application in halogen-free flame retardant polypropylene [J]. Journal of Analytical and Applied Pyrolysis, $111,238-246$.

[11] Cai, Y., Wei, Q., Huang, F. 2008. Preparation and properties studies of halogen-free flame retardant form-stable phase change materials based on paraffin/high density polyethylene composites [J]. Applied Energy, 85, 765-775.

[12] Yong, H., Lifeng, X., Changbo, L. 2014. Static experimental study on flame retardant and explosion suppression performances of fire resistant diesel fuel [J]. Procedia Engineering, 84, 419-426.
[13] Zhao, S., Xu, B., He, L. 2017. Study of a novel co-rotating non-twin screw extruder in processing flame retardant polymer materials [J]. Journal of Polymer Engineering, 37 (8), 01-20.

[14] Feng, C., Liang, M., Jiang, J. 2016. Synergistic effect of a novel triazine charring agent and ammonium polyphosphate on the flame retardant properties of halogen-free flame retardant polypropylene composites [J]. Thermochimica Acta, 627-629, 83-90.

[15] Chen, Y., Wang, W., Qiu, Y. 2017. Terminal group effects of phosphazene-triazine bi-group flame retardant additives in flame retardant polylactic acid composites [J]. Polymer Degradation and Stability, 140, 166-175.

[16] Ni, H.G., Lu, S.Y., Mo, T. 2016. Brominated flame-retardant emissions from the open burning of five plastic wastes and implications for environmental exposure in China [J]. Environmental Pollution, 214, 70-76. 